\title{
Finite Element Simulation of Surface Acoustic Wave Based Sensors
}

\author{
K. Shaposhnikov ${ }^{1}$, P. Nicolay ${ }^{2}$, M. Kaltenbacher ${ }^{1}$ \\ ${ }^{1}$ Institute of Mechanics and Mechatronics, Vienna University of Technology, Vienna, Austria, \\ kirill.shaposhnikov@tuwien.ac.at, manfred.kaltenbacher@tuwien.ac.at \\ ${ }^{2}$ Carinthian Tech Research (CTR AG), Villach, Austria, \\ pascal.nicolay@ctr.at
}

\begin{abstract}
:
We propose a mathematical model for the simulation of surface acoustic wave (SAW) devices. The model describes the propagation of waves in a unit cell of a periodic structure and uses the finite element method (FEM) approach along with the perfectly matched layer (PML) technique. The periodicity is treated by means of the method of Lagrange multipliers. Numerical results demonstrate the accuracy and the applicability of the developed computational scheme.
\end{abstract}

Key words: Surface acoustic waves, finite element method, periodic boundary conditions, harmonic admittance, coupling-of-mode parameters.

\section{Introduction}

Surface acoustic wave based devices are widely used as pressure, temperature, humidity, gas sensors. They consist of a piezoelectric substrate and a grating of electrodes and operate according to the inverse piezoelectric effect. However, the geometry of structure might be quite complex: the substrate and the electrodes can contain several layers, the electrodes can have different shape, and cover layers can be applied to protect a devise operating in a hostile environment. Therefore, accurate and efficient simulation tools are needed to investigate such structures and derive and optimize the desired parameters of the device.

In this work, we propose a model utilizing fullFEM approach for the simulation of wave propagation in periodic piezoelectric structures under periodic electrode gratings.

\section{Mathematical Model}

As an approach to a surface acoustic wave device, we take a structure which main parts are a piezoelectric substrate and metal electrodes located on the upper side of the substrate. The electrodes are supposed to be driven by an external alternating voltage, and therefore mechanical waves arise in the substrate because of the inverse piezoelectric effect.
We make following assumptions concerning the structure:

a. The substrate is much thicker than electrodes, so we suppose that the substrate is infinitely thick and occupies the lower half-space.

b. The electrodes form a periodic grating and their number is infinite.

c. The aperture of the grating, i.e. the size in transversal dimension, is much greater than the height of the electrodes. Therefore, we can neglect the variation of all quantities with respect to that direction and consider the problem in 2D.

d. There are no external forces applied to the structure, and the voltage is harmonic in time with the angular frequency $\omega=2 \pi f$.

These assumptions are common and allow using different mathematical models and numerical methods to make accurate simulations of SAW devices [1], [2].

In this case the process of wave propagation in piezoelectric media is mathematically described by the system of partial differential equations [3]

$\nabla \cdot[\boldsymbol{\sigma}]+\omega^{2} \rho \boldsymbol{u}=0$,

$\nabla \cdot \boldsymbol{D}=0$. 
In (1), (2), $[\boldsymbol{\sigma}]$ and $\boldsymbol{D}$ denote the mechanical stress tensor and the electric field displacement, respectively; $\rho$ is the mass density, and $\boldsymbol{u}$ is the mechanical displacement vector.

The equations (1), (2) are coupled by linearized constitutive equations

$$
\begin{aligned}
& {[\boldsymbol{\sigma}]=\left[\boldsymbol{c}^{E}\right][\boldsymbol{S}]-[\boldsymbol{e}]^{t} E,} \\
& \boldsymbol{D}=[\boldsymbol{e}][\boldsymbol{S}]+\left[\boldsymbol{\varepsilon}^{S}\right] E,
\end{aligned}
$$

where the material data is given by the tensors of mechanical moduli $\left[\boldsymbol{c}^{E}\right]$, electric permittivity $\left[\boldsymbol{\varepsilon}^{S}\right]$, and piezoelectric moduli $[\boldsymbol{e}]$. In their turn, the mechanical strain tensor $[\boldsymbol{S}]$ and the electric field intensity $\boldsymbol{E}$ are determined by the mechanical displacement $\boldsymbol{u}$ and the electric potential $\varphi$ as follows

$$
\begin{aligned}
& {[\boldsymbol{S}]=\frac{1}{2}\left(\nabla \boldsymbol{u}+(\nabla \boldsymbol{u})^{t}\right)=B \boldsymbol{u},} \\
& \boldsymbol{E}=-\nabla \varphi
\end{aligned}
$$

The superscripts " $E$ " and " $S$ " in (3), (4) mean that the values have been taken at constant electric field and mechanical strain, respectively. For the sake of simplicity they are omitted in further expressions.

Next, we substitute $[\boldsymbol{\sigma}]$ and $\boldsymbol{D}$ in (1), (2) for (3), (4). Using (5), (6), we come up with the following system of second order partial differential equations

$$
\begin{aligned}
& B^{t}[\boldsymbol{c}] B \boldsymbol{u}+B^{t}[\mathbf{e}]^{t} \nabla \varphi+\omega^{2} \rho \boldsymbol{u}=0, \\
& \nabla \cdot[\mathbf{e}] B \boldsymbol{u}-\nabla \cdot[\boldsymbol{\varepsilon}] \nabla \varphi=0 .
\end{aligned}
$$

Taking $\boldsymbol{w}=(\boldsymbol{u}, \varphi)$ we can write

$$
\tilde{B}^{t}[\tilde{\boldsymbol{c}}] \tilde{B} \boldsymbol{w}+\omega^{2} \tilde{\rho} \boldsymbol{w}=0
$$

Now, let us refer to the periodicity of the electrode grating. The initial domain, where the equation (7) is given, can be represented as a union of unit cells in such a case. Then the solution $\boldsymbol{w}$ must fulfill the periodic boundary conditions on the left $\Gamma_{L}$ and the right $\Gamma_{R}$.sides of each cell $\Omega$ (see Fig. 1). According to Floquet's theory [4], we obtain

$$
\begin{aligned}
& \left.\boldsymbol{w}\right|_{\Gamma_{\mathrm{R}}}=\left.\theta \boldsymbol{w}\right|_{\Gamma_{\mathrm{L}}}, \\
& \left.\boldsymbol{T}_{n}\right|_{\Gamma_{\mathrm{R}}}=-\left.\theta \boldsymbol{T}_{n}\right|_{\Gamma_{\mathrm{L}}},
\end{aligned}
$$

where $\theta$ is a complex-valued factor of the periodicity, and $\boldsymbol{T}_{n}=[\tilde{\boldsymbol{c}}] \tilde{\mathrm{B}} \boldsymbol{w} \cdot \boldsymbol{n}$.
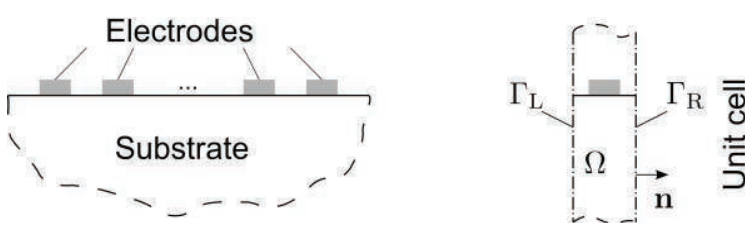

Fig. 1. To the periodic boundary conditions implementation.

The open domain problem is treated here in the same way as in [5]. Namely, we utilize the complex frequency shifted perfectly matched layer (CFS-PML) technique [6]. The PML is designed to damp the waves propagating downward into the substrate, so that we can introduce an artificial lower boundary $\Gamma_{B}$ setting $\boldsymbol{w}=0$ on it.

Another distinctive property of PML is its ability to absorb the waves reflected from $\Gamma_{\mathrm{B}}$ and propagating back to the top, so that the spurious reflections become suppressed.

The numerical scheme for solving the coupled system of partial differential equations (see equation (7)) is based on full-FEM approach. The upper boundary of the structure is assumed to be free of stress, i.e. $\boldsymbol{T}_{n}=0$ on $\Gamma_{U}$. As for periodic boundary conditions (8), (9), they can be incorporated into the numerical model using the method of Lagrange multipliers [7] which is widely applied to the problems related to SAW in periodic structures [2], [5]. The idea is to substitute the multiplier for the surface normal stress on the left or on the right side, e.g. $\boldsymbol{\lambda}=\boldsymbol{T}_{n}$ on $\Gamma_{\mathrm{L}}$.

This method leads to a linear system which can be written in a block form as follows

$$
\left[\begin{array}{cc}
K-\omega^{2} M & (\theta R-L)^{t} \\
R-\theta L & 0
\end{array}\right]\left[\begin{array}{l}
\bar{w} \\
\bar{\lambda}
\end{array}\right]=\left[\begin{array}{l}
\bar{f} \\
0
\end{array}\right]
$$


The system is a saddle point linear system which can be solved using both direct and iterative numerical methods. We also mention that everything described above remains valid in the full 3D case, too. The model based on FEM shows high versatility in this sense.

\section{Results and Discussion}

We utilize the harmonic admittance technique [2] to extract the parameters relevant to the investigation of the structure behavior. The admittance is calculate as follows

$Y=\frac{i \omega}{V} \oint_{\Gamma} q_{\mathrm{e}} d \Gamma$

where $V$ is a given voltage, and $q_{\mathrm{e}}$ is the surface density of electric charge distributed on the electrode surface $\Gamma$.

The harmonic admittance calculated according to (10) allows us to perform the modal analysis of the structure in order to obtain the edges of the stop-band, further extract all necessary parameters of the structure, and study their dependence on the height and the shape of electrodes, metallization ratio, etc.

In fact, the parameters determination based on the harmonic admittance analysis implies the fitting procedure with the admittance used in coupling-of-modes (COM) model [8]. Thereby, the resonant frequency $f_{R}$ is one of the edges of the stop-band in case of a short-circuited grating. For an open-circuited grating, it is the anti-resonant frequency $f_{\mathrm{A}}$. These frequencies are usually clearly visible as poles and zeros on the curves of the harmonic admittance imaginary part. Another edge frequency $f_{\mathrm{C}}$ is sometimes distinguishable as soon as the periodicity is detuned.

We refer to the shifted voltage model [9] (see Fig. 2) and find that the voltage $V_{n}=(-1)^{n} V e^{i \beta p}$ corresponds to the periodicity factor $\theta=-e^{i \beta p}$. Setting $\beta=2 \pi \delta / p$ yields then $\theta(\delta)=-e^{i \cdot 2 \pi \delta}$. The factor $\delta$ is called the detuning coefficient.

As an example we consider the propagation of Rayleigh waves on $\mathrm{YZ}-\mathrm{LiNbO}_{3}$ cut under single electrode grating made of aluminum. The metallization ratio has been taken $50 \%$; the height of the electrodes, as $h / \lambda=1 \%$. The calculated harmonic admittance curves are depicted in Fig. 3. The blue curve corresponds to the case with no detuning. The only visible peaks are at the resonant and anti-resonant frequencies. The green curve is obtained with a small detuning $\delta=5 \cdot 10^{-4}$. The second edge of the stop-band corresponds to the frequency $f_{\mathrm{C}}$.

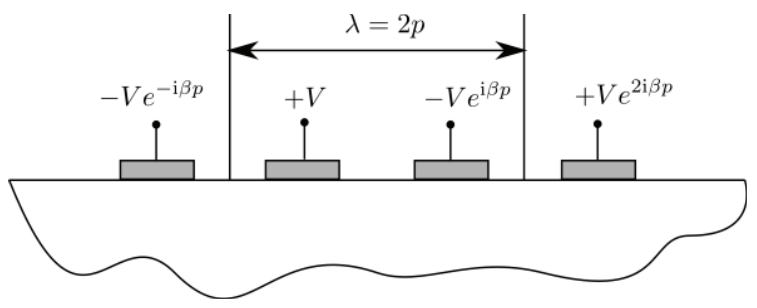

Fig. 2. To the shifted voltage model.

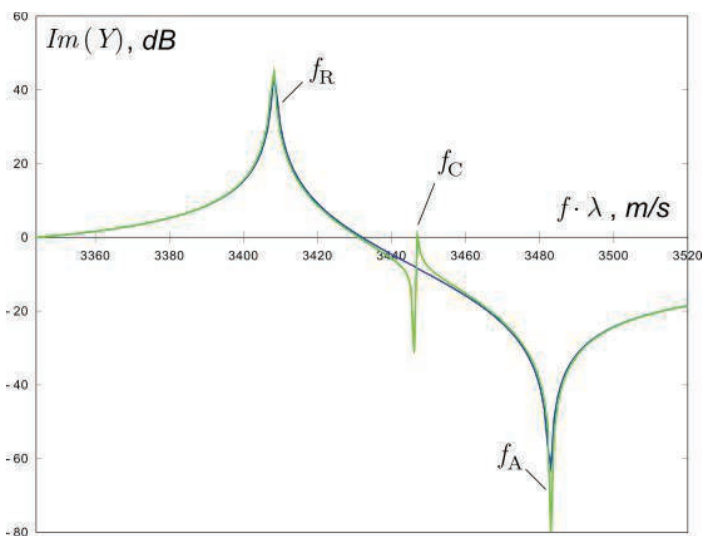

Fig. 3. The curves of the harmonic admittance calculated with and without a detuning.

The dispersion curves obtained for the considered structure at different height of electrodes are shown in Fig. 4.

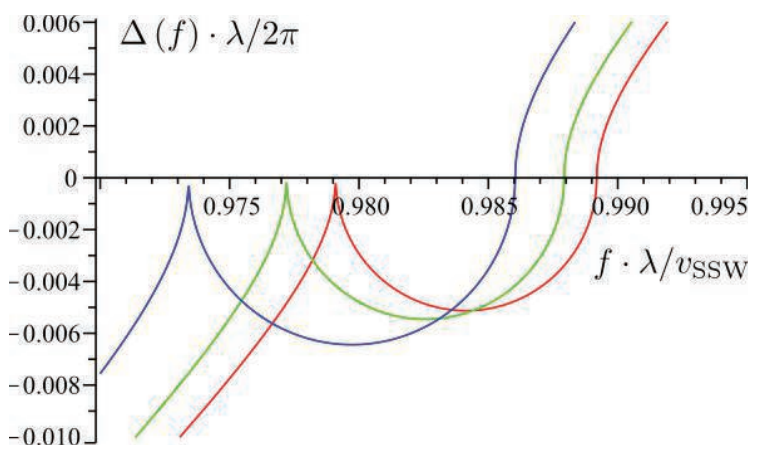

Fig. 4. Dispersion curves for $\mathrm{YZ}-\mathrm{LiNbO}_{3}$ under a short-circuited grating with the height of electrodes $h / \lambda=0,1$, and $2.5 \%$ (red, greed, and blue curves).

Note that the curves in Fig. 4 correlate well with those calculated in [9] using Green's function model.

The models based on a full FEM approach seem the most attractive if one need to simulate a structure with a multi-layered piezoelectric substrate. The efficiency of FEM remains independent of the number of layers, while other methods, such as BEM, can become less effective for multi-layered structures.

We take the substrate consisting of a GaN layer grown on silicon and study how the parameters 
of SAW are influenced by the layer thickness. The electrodes are made of gold and have the height $h / \lambda=2 \%$. The metallization ratio equals to $50 \%$. Fig. 5 shows the shift of the resonant peaks on the harmonic admittance curves. The curves correspond to the following thicknesses of GaN layer: black $-0.02 \lambda$; red $-0.05 \lambda$; green $-0.1 \lambda$; blue $-0.2 \lambda$; cyan $-0.5 \lambda$.

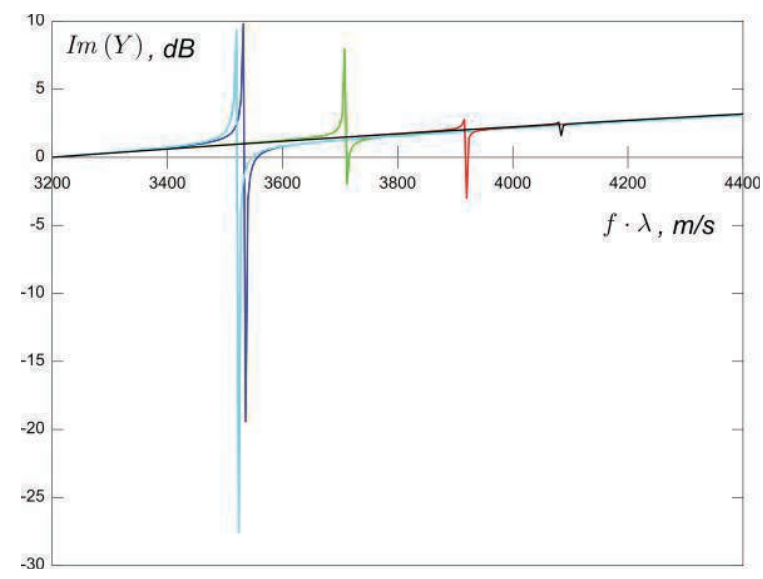

Fig. 5. Harmonic admittance of GaN/Si substrate under gold electrode grating.

The dependences of SAW velocity and the reflection coefficient on the layer thickness are shown in Fig. 6, 7, respectively. One can see that the behavior of the reflection coefficient is not monotonic. The maximum is reached at about $h_{\mathrm{GaN}}=0.75 \lambda$.

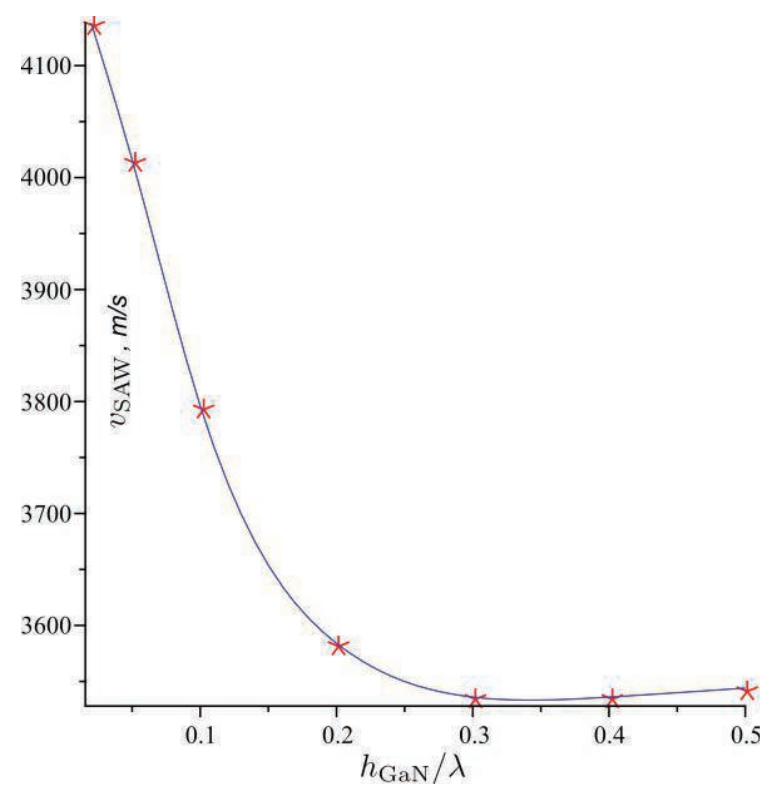

Fig. 6. SAW velocity vs. GaN layer thickness.

The influence of the other parameters, like the metallization ratio, the height and the shape of electrodes, can also be investigated by means of the proposed model.

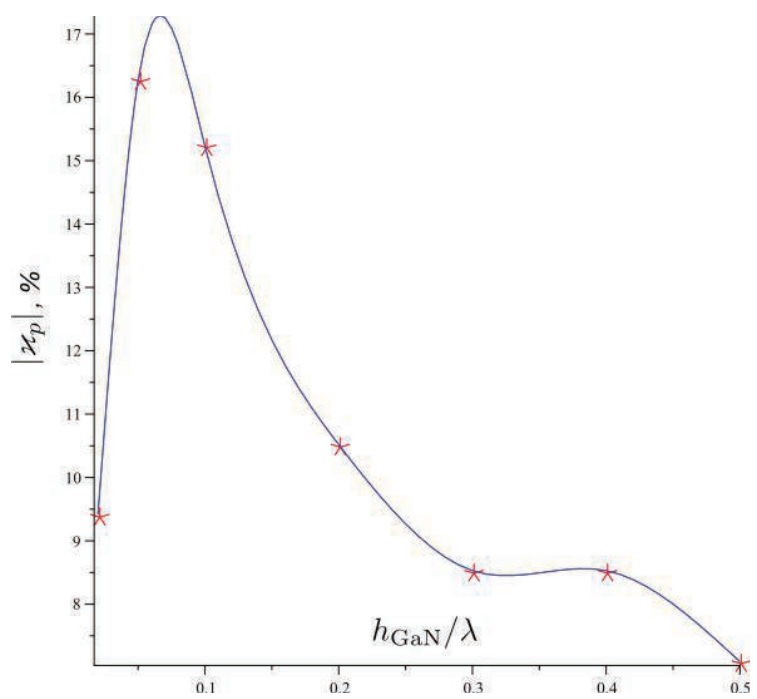

Fig. 7. SAW reflection coefficient vs. GaN layer thickness.

\section{Conclusion}

The model based on full-FEM approach demonstrates high efficiency and reliability for the simulation of SAW devices. The harmonic admittance analysis is applied to extract desired parameters of the structure. The main advantage of the model is due to the robustness of FEM in the problems with complex geometry..

\section{Acknowledgement}

The Competence Centre CTR is funded within the R\&D Program COMET - Competence Centers for Excellent Technologies by the Federal Ministries of Transport, Innovation and Technology (BMVIT), of Economics and Labour (BMWA) and it is managed on their behalf by the Austrian Research Promotion Agency (FFG). The Austrian provinces (Carinthia and Styria) provide additional funding.

\section{References}

[1] V. Laude, A. Reinhardt, M. Wilm, A. Khelif, S. Ballandras, Fast FEM/BEM simulation of SAW devices via asymptotic waveform evaluation, IEEE Trans. UFFC, vol. 51, no. 3, 359-363 (2004); doi: 10.1109/ULTSYM.2004.1417758

[2] M. Hofer, N. Finger, G. Kovacs, J. Schöberl, U. Langer, R. Lerch, Finite element simulation of bulk- and surface acoustic wave interaction in SAW devices, IEEE Ultrason. Symp., 53-56 (2002); doi: 10.1109/ULTSYM.2002.1193351

[3] M. Kaltenbacher, Numerical simulation of mechatronic sensors and actuators, 2nd ed. Springer (2007); doi: 10.1007/978-3-540-71360-9

[4] P. A. Kuchmet, Floquet theory for partial differential equations, Uspekhi Mat. Nauk, vol. 37, no. 4, 3-52 (1982);

doi: 10.1070/RM1982v037n04ABEH003965 
[5] K. Shaposhnikov, M. Kaltenbacher, P. Nicolay, Fast full-FEM computation of COM paremeters. Application to multilayered SAW structures, IEEE Ultrason. Symp., 1501-1504 (2014); doi: 10.1109/ULTSYM.2014.0371

[6] F. H. Drossaert, A. Giannopoulos, A nonsplit complex frequency shifted PML based on recursive integration for FDTD modeling of elastic waves, Geophysics, vol. 72, no. 2, 9-17 (2007); doi: 10.1190/1.2424888

[7] M. Aubertin, T. Hennerson, F. Piriou, P. Guerin, J.-C. Mipo, Periodic and anti-periodic boundary conditions with the lagrange multipliers in FEM, IEEE Trans. Magn., vol. 40, no. 8, 3417-3420 (2010); doi: 10.1109/TMAG.2010.2046723

[8] D.-P. Chen, H. A. Haus, Analysis of metal-strip SAW gratings and transducers, IEEE Trans. Sonics Ultrason., vol. 32, no. 3, 395-408 (1985); doi: 10.1109/T-SU.1985.31609

[9] 1985. J. Koskela, V. P. Plessky, M. M. Salomaa, SAW/LSAW COM parameter extraction from computer experiments with harmonic admittance of a periodic array of electrodes, IEEE Trans. UFFC, vol. 46, no. 4, 806-816 (1999); doi: $10.1109 / 58.775644$ 